\title{
Feasibility of resuscitation contrast-enhanced postmortem computed tomography using cardiopulmonary resuscitation technique with chest compression immediately after death
}

\author{
Kazunori lizuka ${ }^{1 *}$, Namiko Sakamoto ${ }^{2}$, Seiji Shiotani ${ }^{3}$ and Atsushi Komatsuzaki ${ }^{4}$
}

\begin{abstract}
Purpose: Our purpose was to evaluate image delineation ability of contrast-enhanced post-mortem computed tomography (CEPMCT) using cardiopulmonary resuscitation technique of chest compression, named "resuscitation CEPMCT".

Materials and methods: Non-traumatically-deceased 15 subjects (7 men; 8 women) aged 19-87 years (mean 61 years) underwent resuscitation CEPMCT. The contrast-enhanced technique, while injecting $100 \mathrm{ml}$ of contrast media from the right cubital vein at a rate of $1 \mathrm{ml} / \mathrm{s}$, chest compression was performed for 2 minutes at a rate of 100 times/min (a total of 200 times). CT attenuation values (Hounsfield Unit: HU) were measured in 8 target vessels: 1) pulmonary artery, 2) coronary artery, 3) ascending aorta, 4) abdominal aorta, 5) celiac trunk, 6) common iliac artery, 7) superior vena cava, and 8) inferior vena cava. One-sided Student's t-test was performed to assess whether measured values were higher than $140 \mathrm{HU}$ by setting p-value at 0.05 .
\end{abstract}

Results: Measured CT values in the 8 vessels were 1) pulmonary artery: $325 \pm 140 \mathrm{HU}, 2)$ coronary artery: $240 \pm 73$ $\mathrm{HU}, 3)$ ascending aorta: $321 \pm 127 \mathrm{HU}, 4)$ abdominal aorta: $286 \pm 96 \mathrm{HU}, 5)$ celiac trunk: $233 \pm 62 \mathrm{HU}, 6)$ common iliac artery: $260 \pm 114 \mathrm{HU}, 7)$ superior vena cava: $422 \pm 187 \mathrm{HU}$, and 8) inferior vena cava: $301 \pm 142 \mathrm{HU}$, showing significantly higher values than the threshold value of $140 \mathrm{HU}$. Resuscitation CEPMCT detected one case of pulmonary arterial thromboemboli death.

Conclusion: Resuscitation CEPMCT using chest compression immediately after death has the possibility of detecting thromboembolus in major vessels, despite the simplicity of the technique.

Keywords: Post-mortem computed tomography (PMCT); Contrast-enhanced PMCT (CEPMCT); Cardiopulmonary resuscitation (CPR); Chest compression; $\mathrm{CT}$ attenuation value

\section{Introduction}

Due to the worldwide decline in conventional autopsy rates, the need for and frequency of post-mortem crosssectional imaging as a complementary, supplementary or alternative method for autopsy have increased worldwide (Brogdon 1998; Swift and Rutty 2006; Oesterhelweg and Thali 2009; Rutty et al. 2012a).

\footnotetext{
* Correspondence: nori5491@gmail.com

'Department of Radiology, National Hospital Organization Matsumoto Medical Center, 2-20-30 muraimachiminami, Matsumoto, Nagano 399-8701, Japan

Full list of author information is available at the end of the article
}

Death cause detection rate of non-traumatic PMCT alone is approximately $30 \%$ in general, while PMCT is especially useful in showing fatal hemorrhagic lesions, including cerebral/subarachnoid hemorrhage, aortic dissection, and aortic aneurysmal rupture (Kaneko et al. 2010; Takahashi et al. 2012; Okuda et al. 2013). Thromboembolism of the coronary artery or pulmonary artery is difficult to detect with PMCT; however, contrast-enhanced PMCT (CEPMCT) can detect them (Jackowski et al. 2005; Grabherr et al. 2007; Steffen et al. 2008; Sakamoto et al. 2009; Iizuka et al. 2009; Kikuchi et al. 2010; Ross et al. 2011; Jolibert et al. 2011; Saunders 
et al. 2011; Roberts et al. 2011; Ross et al. 2012; Rutty et al. 2012b). Several methods of CEPMCT have been developed worldwide. In Western countries, surgical management for embalming is necessary to perform CEPMCT (Morgan et al. 2014). In Japan, CEPMCT is performed by injecting contrast media from the venous route in combination with chest compression. This technique is named "Resuscitation CEPMCT" because the method is similar to that of the cardiopulmonary resuscitation technique (Okuda et al. 2013; Sakamoto et al. 2009; Iizuka et al. 2009; Kikuchi et al. 2010); however, no literature has been published regarding quantitative evaluation of the vascular delineation ability. Herein, we report the image delineation ability of Japanese CEPMCT technique with chest compression, based on retrospectively measured $\mathrm{CT}$ attenuation values in major vessels.

\section{Materials and methods Subjects}

Our subjects were 15 non-traumatically-deceased patients (7 men and 8 women) aged 19-87 years (mean 61 years) who underwent resuscitation CEPMCT with chest compression between September 2009 and March 2010. Each death was confirmed after subject's arrival in a state of cardiopulmonary arrest at the emergency room (ER) of National Hospital Organization Tokyo Medical Center. Cardiopulmonary resuscitation (CPR) was performed on all subjects by emergency technicians during transport and in the ER by emergency medical physicians for $30 \mathrm{~min}$. in accordance with the 2010 American Heart Association (AHA) Guidelines for CPR (American Heart Association 2010). CPR included continuous external chest compression, artificial respiration with bag-valve mask ventilation following endotracheal intubation, electric defibrillation, peripheral intravenous catheterization following administration of epinephrine at $1 \mathrm{mg}$, and infusion. Although the family of each subject did not consent to autopsy, they did consent to PMCT and resuscitation CEPMCT. Causes of death were diagnosed based on a comprehensive evaluation of a subjects' history of present illness, medical history, laboratory results, and PMCT findings, which included aortic dissection (4 cases), cerebral hemorrhage (3 cases), ischemic heart disease (2 cases), pneumonia (2 cases), gastric cancer (1 case), drug toxicity (1 case), and suffocation (1 case) and pulmonary thromboembolism (1 case).

\section{Methods}

Firstly, PMCT was performed immediately after the confirmation of death using a clinical scanner in the Radiology Department of National Hospital Organization Tokyo Medical Center, with prior approval of the institutional review board. PMCT was performed with an 8-channel multidetector-row CT scanner (Lightspeed Ultra; GE Healthcare, Milwaukee, USA). The imaging parameters for the head, neck, thorax, abdomen, and pelvis were determined for helical scan mode with settings of auto $\mathrm{mA}$ (200-400, noise index: 6.0), $120 \mathrm{kV}, 0.5 \mathrm{sec} /$ rotation, $1.25 \mathrm{~mm}$ collimation, 1.625 pitch, scan speed of $16.75 \mathrm{~mm} /$ rotation, and helical thickness of $5 \mathrm{~mm}$.

Secondly, resuscitation CEPMCT was performed as described below. For injecting a contrast media from the right cubital vein, an automatic injector (Dual Shot GX, Nemoto Kyorindo Inc., Japan) was used for the peripheral intravenous catheter retained for infusion during CPR. The contrast media used was iopamidol (Oypalomin 300 Injection Syringe, Konica Minolta Holdings Inc., Japan), which is a non-ionic media generally used in clinical practice. A dose amount of $100 \mathrm{ml}$ was injected at a rate of $1 \mathrm{ml} /$ second. While injecting the contrast media, chest compression was done on the CT table for 2 minutes at a rate of 100 times/ minutes (a total of 200 times), in accordance with 2010
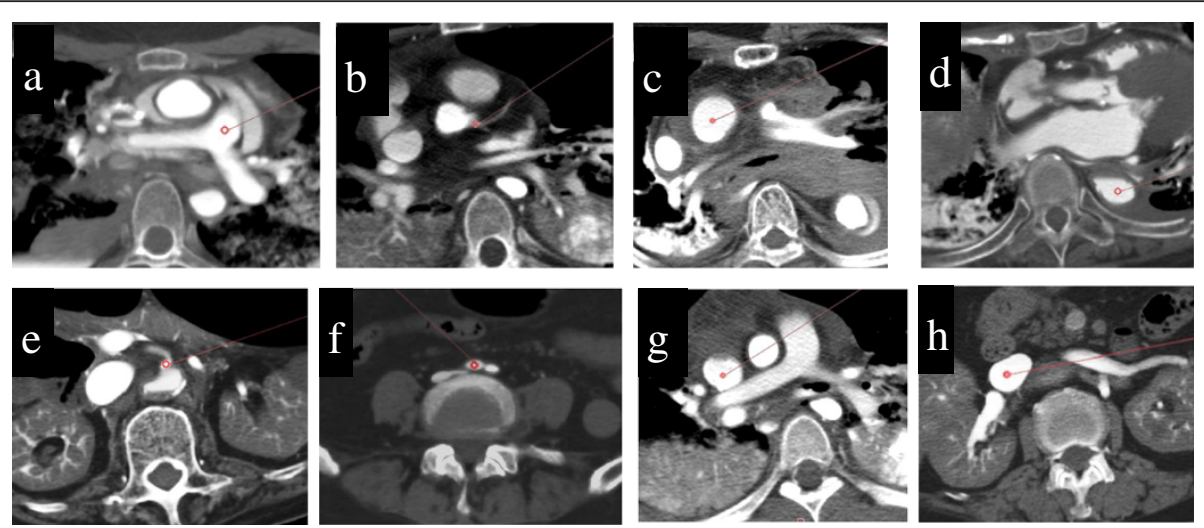

Figure 1 PMCT of 8 target blood vessels of a 64-year-old man. A red circle indicates a region of interest in which $C T$ attenuation value (HU) was measured. a. Pulmonary artery trunk $\mathbf{b}$. Left main coronary artery $\mathbf{c}$. Ascending aorta at the level of the tracheal bifurcation $\mathbf{d}$. Abdominal aorta at the level of the diaphragm e. Root of the celiac trunk $\mathbf{f}$. Right common iliac artery immediately after branching from the descending aorta $\mathbf{g}$. Superior vena cava at the level of bifurcation pulmonary artery. $\mathbf{h}$. Inferior vena cava at the level of renal vein. 
Table 1 CT attenuation values measured on contrast-enhanced postmortem CT

\begin{tabular}{lcccccccccccccccc}
\hline & \multicolumn{11}{c}{ Case I.D. No. } \\
\cline { 2 - 5 } & $\mathbf{1}$ & $\mathbf{2}$ & $\mathbf{3}$ & $\mathbf{4}$ & $\mathbf{5}$ & $\mathbf{6}$ & $\mathbf{7}$ & $\mathbf{8}$ & $\mathbf{9}$ & $\mathbf{1 0}$ & $\mathbf{1 1}$ & $\mathbf{1 2}$ & $\mathbf{1 3}$ & $\mathbf{1 4}$ & $\mathbf{1 5}$ & Mean \pm SD \\
\hline 1. Pulmonary artery & 559 & 186 & 234 & 516 & 215 & 341 & 216 & 290 & 371 & 198 & 481 & 234 & 191 & 569 & 269 & $325 \pm 140^{*}$ \\
2. Coronary artery & 350 & 265 & 286 & 205 & 198 & 271 & 168 & 226 & 291 & 226 & 210 & 254 & 165 & 388 & 100 & $240 \pm 73^{*}$ \\
3. Ascending aorta & 425 & 310 & 358 & 359 & 221 & 492 & 224 & 317 & 429 & 204 & 335 & 249 & 191 & 593 & 114 & $321 \pm 127^{*}$ \\
4. Abdominal aorta & 430 & 303 & 346 & 369 & 201 & 303 & 222 & 300 & 355 & 198 & 265 & 258 & 190 & 453 & 97 & $286 \pm 96^{*}$ \\
5. Celiac artery & 305 & 241 & 258 & 220 & 189 & 245 & 223 & 289 & 303 & 189 & 220 & 264 & 194 & 287 & 61 & $233 \pm 62^{*}$ \\
6. Common iliac artery & 413 & 235 & 365 & 173 & 182 & 517 & 228 & 280 & 358 & 220 & 231 & 284 & 171 & 187 & 58 & $260 \pm 114^{*}$ \\
7. Superior vena cava & 823 & 236 & 218 & 684 & 241 & 335 & 226 & 447 & 548 & 455 & 628 & 503 & 275 & 420 & 290 & $422 \pm 187^{*}$ \\
8. Inferior vena cava & 625 & 240 & 214 & 624 & 216 & 279 & 215 & 215 & 333 & 208 & 368 & 230 & 167 & 287 & 298 & $301 \pm 142^{*}$ \\
\hline
\end{tabular}

Notes: $\mathrm{CT}$ attenuation values are shown as Hounsfield Unit (HU).

*Significantly greater CT attenuation value than a threshold of $140 \mathrm{HU}(p<0.05)$.

AHA Guidelines for CPR (American Heart Association 2010). Scanning parameters for resuscitation CEPMCT were the same as for PMCT.

Intra-luminal CT attenuation values of the following 8 vessels were measured: 1) pulmonary artery trunk (Figure 1a); 2) left main coronary artery (Figure 1b); 3) ascending aorta at the level of the tracheal bifurcation (Figure 1c); 4) abdominal aorta at the level of the diaphragm (Figure 1d); 5) root of the celiac trunk (Figure 1e); 6) right common iliac artery immediately after branching from the descending aorta (Figure 1f); 7) superior vena cava at the level of bifurcation pulmonary artery (Figure 1g); and 8) inferior vena cava at the level of renal vein (Figure 1h). A circle-shaped region of interest (ROI) with the diameter of $3 \mathrm{~mm}$ was placed in the center of the subject vessels, except for the 2) left main coronary artery and 5) root of the celiac trunk, where the diameter of the ROI was set at $1 \mathrm{~mm}$.

A threshold value setting for detection of thromboemboli For detection of thromboemboli, a threshold value of 140 Hounsfield Unit (HU) was chosen as the minimallynecessary enhancement value in the vessel. Generally, $\mathrm{CT}$ attenuation value of thrombus is $50-80 \mathrm{HU}$, plaque (mainly fat and fiber) of the coronary artery is less than
$120 \mathrm{HU}$, and plaque (mainly calcification) of the coronary artery is higher than $120 \mathrm{HU}$ (Schroeder et al. 2004). The contrast discrimination threshold (namely, CT value difference by which radiologists never fails to distinguish among tissues) is approximately $20 \mathrm{HU}$ (Ariji et al. 1993). Therefore, theoretically, thrombus without including calcification can be detected when CT attenuation value in the vessel is higher than $140 \mathrm{HU}$.

The one-sided $t$ test was used to evaluate whether the mean attenuation value of each target vessel was significantly greater than $140 \mathrm{HU}$. A P value of less than 0.05 was considered to be a statistically significant difference.

\section{Results}

Mean CT attenuation values of each targeted vessel are shown in Table 1. All of the 8 examined vessels had CT attenuation values of greater than $140 \mathrm{HU}$. There was no extravasation of contrast media due to postmortem increased permeability of the vascular wall. In one case having thromboemboli of the pulmonary artery, detection of the thrombi was difficult with PMCT (Figure 2a). On a resuscitation CEPMCT image, CT value of the pulmonary arterial trunk was $269 \mathrm{HU}$, and that of thromboemboli in the pulmonary artery was 54 HU. The filling defects in the pulmonary artery indicated the presence of thromboemboli (Figure 2b).

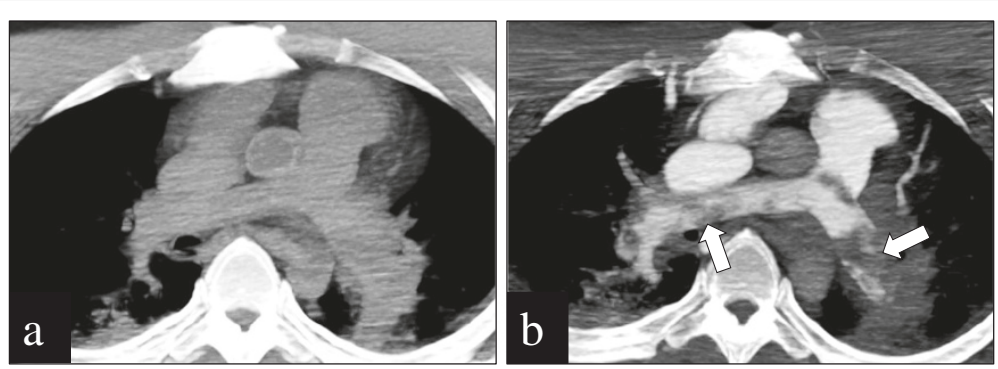

Figure 2 A 38-year-old man died for thromboembolism of pulmonary artery. Detection of thrombi is difficult with PMCT (a). On a resuscitation CEPMCT image, CT value of the pulmonary arterial trunk was $269 \mathrm{HU}$, and that of thromboemboli in the pulmonary artery was 54 $\mathrm{HU}$. The filling defects in the pulmonary artery indicated the presence of thromboemboli (b). 


\section{Discussion}

The resuscitation CEPMCT technique in the present study using intravenous contrast media injection and chest compression delineated all 8 vessels with $\mathrm{CT}$ attenuation values of over $140 \mathrm{HU}$. A small thrombus might be difficult to detect using enhanced $\mathrm{CT}$, and the negative detection rate of acute pulmonary embolism is reported to be $60 \%$ using multidetector CT (Stein et al. 2006). However, CEPMCT is considered capable of detecting relatively large thromboemboli which can be a cause of sudden death. In our study, thrombi were detected in one case of pulmonary arterial thromboemboli death. The formation of postmortem blood clots is closely linked to the length of the agonal interval (Ross et al. 2012). In the case of a sudden death, plasminogen activator is released into the vessel, resulting in increased fluidity of blood (Shiotani et al. 2002). Therefore, PMCT performed immediately after death can differentiate thromboemboli from postmortem coagulation. This infers the feasibility of resuscitation CEPMCT for detection of thromboemboli at least in the pulmonary arteries, which has been difficult to do with PMCT alone.

Chest compression during CPR increases blood pressure, and generates cardiac output of approximately one-third to one-fourth of the normal state of a living body (Jackson and Freeman 1983; Paradis et al. 1989). This phenomenon enables delineation of our resuscitation CEPMCT with chest compression. When chest compression is not applied for CEPMCT, injected contrast media migrates from the right atrium to the inferior vena cava, without flowing into the right chamber or left heart (Morgan et al. 2014). With chest compression, contrast media injected from the upper arm (cubital vein) enters the right atrium, moves into the right ventricle, pulmonary artery, pulmonary vein, left atrium, left ventricle, aorta, with the enhanced image of the arterial route being delineated.

There are two drawbacks to this study. One is that all of the subjects were non-traumatically deceased patients. In traumatic cases where chest compression is not effective, such as multiple rib fracture and significant loss of blood volume from bleeding, contrast-enhancement would be insufficient due to the lack of effective perfusion of the contrast media in the body. Another drawback is that it is uncertain whether the injected amounts, concentrations, and flow rate of the contrast media and the time of chest compression we performed were optimal for resuscitation CEPMCT.

We surmise that increased intravascular CT values would be obtained with increased amounts and concentrations of contrast media. On the other hand, an excessive number of chest compressions will widely diffuse contrast media in the body, which may obscure intravascular $\mathrm{CT}$ values. The optimum imaging conditions should be further investigated.
In conclusion, resuscitation CEPMCT with the combination of contrast media injection and chest compression immediately after death is believed to be a relatively simple additional technique to PMCT for the detection of vascular disease-related causes of death.

\section{Availability and requirements}

- Project name: none

- Project home page: none

- Operating system(s): none

- Programming language: none

- Other requirements: none

- License: none

- Any restrictions to use by non-academics: none

Competing interests

The authors declare that they have no competing interests.

\section{Authors' contribution}

$\mathrm{KI}, \mathrm{NS}, \mathrm{SS}$, and AK conceived and designed the study. KI, NS, and AK acquired the data. All authors analysed and interpreted the data. KI drafted the manuscript. NS and SS revised the manuscript. All authors read and approved the final manuscript.

\section{Author details}

'Department of Radiology, National Hospital Organization Matsumoto Medical Center, 2-20-30 muraimachiminami, Matsumoto, Nagano 399-8701, Japan. ${ }^{2}$ Department of Forensic Medicine, Hirosaki University Graduate School of Medicine, Hirosaki, Japan. ${ }^{3}$ Department of Radiology, Tsukuba Medical Center, Tsukuba, Japan. ${ }^{4}$ Department of Radiology, National Hospital Organization Tokyo Medical Center, Meguro, Japan.

Received: 6 December 2013 Accepted: 6 December 2013 Published: 10 December 2013

\section{References}

American Heart Association (2010) Advanced cardiovascular life support. In: Hazinski MF (ed) Highlights of the 2010 American Heart Association Guidelines for CPR and ECC, 1st edn. American Heart Association, Dallas, pp 13-16

Ariji Y, Ariji E, Yoshiura K, Kanda S (1993) A study on contrast discrimination of CT images. Oral Radiol 9:9-16

Brogdon BG (1998) Research and applications of the new modalities. In: Brogdon BG (ed) Forensic Radiology, 1st edn. CBC Press, Boca Raton, pp 333-338

Grabherr S, Djonov V, Yen K, Thali MJ, Dirnhofer R (2007) Postmortem angiography: review of former and current methods. AJR Am J Roentgenol 188:832-838

lizuka K, Sakamoto M, Kawasaki H, Miyoshi T, Komatsuzaki A, Kikuchi S (2009) Examination of the usefulness of the contrast-enhanced Post mortem CT imaging diagnosis. Innervision 24:89-92 (in Japanese)

Jackowski C, Sonnenschein M, Thali MJ, Aghayev E, Von Allmen G, Yen K et al (2005) Virtopsy: postmortem minimally invasive angiography using cross section techniques -implementation and preliminary results. J Forensic Sci 50:1175-1186

Jackson RE, Freeman SB (1983) Hemodynamics of cardiac massage. Emerg Med Clin North Am 1:501-513

Jolibert M, Cohen F, Bartoli C, Boval C, Vidal V, Gaubert JY et al (2011) Angioscanner post-mortem : faisabilité de l'abord artériel sous guidage échographique. J Radiol 92:446-449 (in French)

Kaneko T, Hibi M, Ishibashi M, Nakatsuka A, Omori Y, Ishikura K et al (2010) Postmortem computed tomography is an informative approach for prevention of sudden unexpected natural death in the elderly. Risk Manag Healthc Policy 3:13-20

Kikuchi H, Kawahara K, Tsuji C, Tajima Y, Kuramoto T, Shihara M et al (2010) Post mortem contrast-enhanced computed tomography in a case of sudden death from acute pulmonary thromboembolism. Exp Ther Med 1:503-505 
Morgan B, Sakamoto N, Shiotani S, Grabher S (2014) Postmortem computed tomography (PMCT) scanning with angiography (PMCTA): a description of three distinct methods. In: Rutty GN (ed) Essentials of autopsy practice, 1st edn. Springer-Verlag, London, pp 1-21

Oesterhelweg L, Thali MJ (2009) Experiences with virtual autopsy approach worldwide. In: Thali MJ, Dirnhofer R, Vock P (eds) The virtopsy approach, 1st edn. CRC Press, Boca Raton, pp 475-477

Okuda T, Shiotani S, Sakamoto N, Kobayashi T (2013) Background and current status of postmortem imaging in Japan: short history of "Autopsy imaging (Ai)". Forensic Sci Int 225:3-8

Paradis NA, Martin GB, Goetting MG, Rosenberg JM, Rivers EP, Appleton TJ et al (1989) Simultaneous aortic, jugular bulb, and right atrial pressures during cardiopulmonary resuscitation in humans. Insights into mechanisms. Circulation 80:361-368

Roberts IS, Benamore RE, Peebles C, Roobottom C, Traill ZC (2011) Diagnosis of coronary artery disease using minimally invasive autopsy: evaluation of a novel method of post-mortem coronary angiography. Clin Radiol 66:645-650

Ross SG, Flach PM, Thali MJ (2011) Postmortem angiography. In: Thali MJ, Viner MD, Brogdon BG (eds) Forensic Radiology. CRC Press, Boca Raton, pp 449-459

Ross SG, Thali MJ, Bolliger S, Germerott T, Ruder TD, Flach PM (2012) Sudden death after chest pain: feasibility of virtual autopsy with postmortem CT angiography and biopsy. Radiology 264:250-259

Rutty GN, Brogdon G, Dedouit F, Grabherr S, Hatch GM, Jackowski G et al (2012a) Terminology used in publications for post-mortem cross-sectional imaging. Int J Legal Med 127:465-466

Rutty G, Saunders S, Morgan B, Raj V (2012b) Targeted cardiac post-mortem computed tomography angiography: a pictorial review. Forensic Sci Med Pathol 8:40-47

Sakamoto N, Senoo S, Kamimura Y, Uemura K (2009) Cardiopulmonary arrest on arrival case which underwent contrast-enhanced postmortem CT. J of Jpn Assoc for Acute Med 30:114-115 (in Japanese)

Saunders SL, Morgan B, Raj V, Robinson CE, Rutty GN (2011) Targeted post-mortem computed tomography cardiac angiography: proof of concept. Int I Legal Med 125:609-616

Schroeder S, Kuettner A, Leitritz M, Janzen J, Kopp AF, Herdeg C et al (2004) Reliability of differentiating human coronary plaque morphology using contrast-enhanced multislice spiral computed tomography: a comparison with histology. J Comput Assist Tomogr 28:449-454

Shiotani S, Kohno M, Ohashi N, Yamazaki K, Itai Y (2002) Postmortem intravascular high-density fluid level (hypostasis): CT findings. J Comput Assist Tomogr 26:892-893

Steffen R, Danny S, Stephan B, Andreas C, Lars O, Silke G et al (2008) Postmortem whole-body $C T$ angiography: evaluation of two contrast media solutions. AJR Am J Roentgenol 190:1380-1389

Stein PD, Fowler SE, Goodman LR, Gottschalk A, Hales CA, Hull RD et al (2006) Multidetector computed tomography for acute pulmonary embolism. N Engl J Med 354:2317-2327

Swift B, Rutty GN (2006) Recent advances in postmortem forensic radiology: $C T$ and MRI applications. Open Forensic Sci J 4:355-404

Takahashi N, Higuchi T, Shiotani M, Hirose Y, Shibuya H, Yamanouchi H et al (2012) The effectiveness of postmortem multidetector computed tomography in the detection of fatal findings related to cause of non-traumatic death in the emergency department. Eur Radiol 22:152-160

\section{Submit your manuscript to a SpringerOpen ${ }^{\circ}$ journal and benefit from:}

- Convenient online submission

Rigorous peer review

- Immediate publication on acceptance

- Open access: articles freely available online

- High visibility within the field

- Retaining the copyright to your article

Submit your next manuscript at $\mathbf{s p r i n g e r o p e n . c o m ~}$ 